\title{
Peak-to-average power ratio reduction in wavelet packet modulation using binary phase sequence
}

\author{
Vijaya M. Kulkarni, A. S. Bhalchandra \\ Department of Electronics and Telecommunication, Government Engineering College, Aurangabad (M.S.), India
}

\begin{tabular}{l} 
Article Info \\
\hline Article history: \\
Received Sep 16, 2018 \\
Revised Nov 17, 2018 \\
Accepted Feb 22, 2019 \\
\hline
\end{tabular}

\section{Keywords:}

Complementary cumulative distribution function (CCDF) OFDM

Peak to average power ratio (PAPR)

SLM technique

Wavelet packet modulation (WPM)

\begin{abstract}
A novel method of Peak-to-Average-Power-Ratio reduction is presented in this paper using WPT for multi carrier modulation and Riemann matrix for phase sequencing in SLM. Wavelet Packet Multicarrier Modulation (WPM) is a better alternative to the existing multicarrier transmission system, OFDM system which suffers from high PAPR. Further reduction in Peak to Average Power Ratio (PAPR) is achieved by using Wavelet Packet Modulation (WPM) with SLM technique. Classical SLM uses random phases for phase rotations which suffers from the additional load of transmitting SI (side Information) to receiver. So to overcome this drawback a novel method is proposed here which uses the principle of varying the phases of the subcarrier data block to be transmitted, using rows of the normalised Reimann matrix as phase sequence vectors for the Selected Mapping (SLM) Technique. The generated WPM frame with minimum PAPR is selected and transmitted. The proposed system achieves a significant improvement in PAPR reduction over the OFDM based classical SLM and WPM based classical SLM.
\end{abstract} All rights reserved.

Corresponding Author:

Vijaya M. Kulkarni,

Department of Electronics and Telecommunication,

Government Engineering College,

Aurangabad (M.S.), India.

Email: vijayamkulkarni@gmail.com

\section{INTRODUCTION}

Orthogonal frequency division multiplexing (OFDM) is extensively used and well known high-data rate transmission technology. It is widely used in Digital Television (DTV), Digital Audio Broadcasting (DAB), Digital Subscriber Line (DSL), broadband internet access, wireless networks, power line networks and $4 \mathrm{G}$ mobile communications. It has several advantages such as spectrum efficiency, more resistant to frequency selective fading, greater immunity to noise and simple channel equalization [1], [2]. But OFDM system has a major drawback of high peak-to-average power ratio (PAPR) because of its structure. However the high PAPR reduces the power efficiency and leads to in-band and out-of-band distortions. Lot of approaches have been proposed such as Tone Reservation (TR) and Tone Injection (TI), clipping filtering, coding schemes phase optimization, Partial Transmission Sequence (PTS), nonlinear companding technique and Selective Mapping (SLM), Active Constellation Extension for PAPR reduction [3]-[10]. Embedding side information (SI) index into the original data results in recovering data at the receiver which also helped in reducing the inter-carrier interference (ICI) and narrowband interference(NBI) [11], [12]. In order to overcome the high side lobes in the OFDM system a novel technique called F-OFDM is proposed. It uses the filtering to reduce the out of band emission (OOBE) for efficient spectrum utilization. In this method the block coding such as Arithmetic coding and Huffman coding is used to reduce the PAPR [13]. In the recent years, many evolutionary algorithms have been considered by many researchers for reducing the PAPR. 
One such novel idea of self-adaptive multiplication differential evolution algorithm for optimizing the phase factor rotation in PTS system is proposed [14]. Another evolutionary algorithm is proposed which utilizes the data detection algorithm to improve the computational efficiency. It is an iterative algorithm which contains initialization, preprocessing and equalization steps to minimize impulse noise and achieve low performance loss [15].

In traditional OFDM system, cyclic prefix is used to counter act the effect of inter symbol interference (ISI), but it reduces the spectral efficiency. To overcome these limitations of OFDM system, Wavelet Packet Transform based system is used to achieve low PAPR. Wavelet Packet Modulation have very lower side lobes in transmitted signals which is helpful in reducing intercarrier interference (ICI) and narrowband interference (NBI). Wavelet packet based system does not require any cyclic prefix and hence increases the efficiency. More over Wavelet Packet Modulation is multicarrier modulation based on wavelet packet transform (WPT), which uses orthogonality property of wavelet filter. In wavelet packet transform there is no loss of information, because the decomposition of the time domain signal into different frequency bands is obtained by passing it through successive high pass as well as low pass filters increasing its resolution. Whereas in DWT the decomposition takes place bypassing the signal only through low pass filter. Lot of work has been done by many researchers in reducing peak to average power by using wavelet packet modulation: A.B.Aichaet et al, have used Daubechies family and compared with other wavelet families in Wavelet Packet Division Multiplexing (WPDM) systems [16]. M. Gautier, C. Lereau et et al investigated the, the influence of appropriate choice of pulse shaping characteristics on PAPR reduction in WPM [17]. Volkan Kumbasar, O־guzKucur have suggested a search algorithm for better wavelet packet tree [18] for fixed number of subcarriers using BPSK modulation to reduce PAPR in wavelet packet modulation scheme.Rostamzadeh,Vakily have used distortionless techniques SLM and PTS to reduce PAPR in Wavelet Packet Division Multiplexing (WPDM) [19]. Mohan Baro, Jacek IIow have improved PAPR reduction over traditional OFDM by using wavelet packet preprocessing of the quadrature amplitude modulation [20]. Berna Torunet et al, [21] have used WPM with adaptive phase selection for subcarriers of multicarrier system. Suma et al, have improved PAPR reduction in OFDM by best tree selection using coded discrete cosine harmonic wavelet packet transform [22]. Zakaria, has proposed embedding side information data which has proved to have better bit error ratio (BER) as compared to other renowned schemes [23].

The problem with the above mentioned approaches is that, they are complex and have not achieved PAPR reduction up to the required level. With this framework, a concept of using WPM based SLM method for PAPR reduction is proposed here. Classical SLM uses random phases for phase rotations, which inturn suffers from a disadvantage of sending the side information (SI) from transmitter and causes spectral inefficiency. Various phase sequences like Neumann phase sequence, Hadamard matrix, Chaotic phase sequence are also used [24]-[26]. But PAPR reduction is not up to satisfactory level. In our work, an attempt has been made to use the sequence of phase vectors based on Riemann matrix in SLM technique for improvement in PAPR reduction. By using phase sequences from Riemann matrx, there is no need to transmit the side information from transmitter which conserves the bandwidth.

\section{OFDM SYSTEM AND SELECTED MAPPING(SLM) TECHNIQUE AND WAVELET PACKET MODULATION (WPM)}

\subsection{OFDM System and Peak to Average Power Reduction (PAPR) Definition}

Though OFDM is one of the promising technologies for high data rate transmission, it suffers from high PAPR at the transmitter. The high PAPR distorts the signal when OFDM is applied to power amplifier. So recently a technique in [27] is proposed in which a good PAPR reduction is achieved without signal distortion.

The multicarrier based OFDM system divides available spectrum into number of orthogonal parallel subcarriers which are converted to constellation streams (BPSK/QPSK). These constellation streams are applied to serial to parallel converter to obtain the parallel bit streams $X_{k}=\left[X_{0}, X_{1}, X_{2} \ldots X_{N-1}\right]$. All the streams are of length $\mathrm{N}$. This parallel bit stream data is applied to IFFT to generate the OFDM symbols containing $\mathrm{N}$ parallel data streams having the same bandwidth. The subcarrier frequencies in OFDM are separated by 1/T which results in zero signal distortions while transmission is the signaling interval of OFDM symbol. The output of IFFT generated OFDM symbol can be expressed as

$$
x_{n}=\frac{1}{\sqrt{N}} \sum_{k=0}^{N-1} X_{k} e^{j 2 \pi n k / L N}, n=0,1 \ldots N
$$

Where $j=\sqrt{ }-1, L \geq 1$ is oversampling factor. The PAPR of the transmitted signal in (1), is the ratio of the maximum power to the average power and is expressed as: 


$$
P A P R=\frac{P_{\text {peak }}}{P_{\text {average }}}
$$

$=10 \log _{10}\left(\operatorname{Max}\left[\left|\mathrm{x}_{\mathrm{n}}\right|^{2}\right] / \mathrm{E}\left[\left|\mathrm{x}_{\mathrm{n}}\right|^{2}\right]\right)$.

Where E [.] represents ensemble average, $P_{\text {peak }}$ represents maximum instantaneous power and $P_{\text {average }}$ represents average power.

CCDF of a PAPR is the probability that the PAPR of the data exceeds a certain level.This criterion is used to analyze the PAPR of OFDM signal and is expressed as:

$P A P R=\operatorname{prob}\left(\mathrm{PAPR}>\mathrm{PAPR}_{0}\right)=1-\left(1-\exp \left(\mathrm{PAPR}_{0}\right)\right)^{\mathrm{N}}$

$P A P R_{0}$ denotes the PAPR threshold in $\mathrm{db}$

\subsection{The SLM Technique}

In SLM technique, each OFDM symbol frame is representing the same information after getting mapped to number of $U$ (independent) candidates. Candidates are generated by multiplying sub carriers with $\mathrm{U}$ phase vectors and the one with minimum PAPR is selected. Number of phase sequences U and design of phase sequences are major factors having effect upon PAPR reduction using SLM technique [1].

Classical SLM technique uses randomly generated phase sequences from set $[+1,-1,+\mathrm{j},-\mathrm{j}][9]$ as shown in Figure 1. $\mathrm{X}$ is the modulated data block, $\mathrm{B}^{(\mathrm{U})}$ are the phase sequences, $\mathrm{X}^{(\mathrm{U})}$ are the modified data blocks in the frequency domain. $\mathrm{x}^{(\mathrm{U})}$ are the corresponding equivalent time domain blocks [1] and is represented as

$$
x_{u}(t)=\sum X_{k} B_{u} e^{j 2 \pi k \Delta f t} \quad, 0 \leq t \leq N T
$$

where $\mathrm{k}$ is changing from 0 to $\mathrm{N}-1, \mathrm{u}=1,2 \ldots \mathrm{U}, \mathrm{N}$ represents both: number of subcarriers and length of $\mathrm{X}$ and from these $\mathrm{X}^{(\mathrm{U})}$, modified data block with the lowest PAPR is selected for transmission [1].

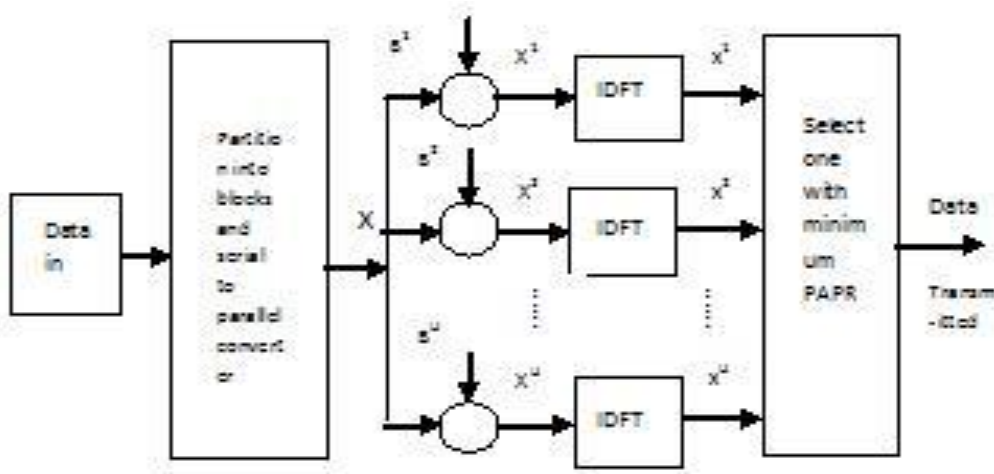

Figure1. Selected mapping technique [1]

\subsection{Riemann Matrix Phase Sequence Set Generation}

Riemann matrix (R) is used to provide phase sequence set. In the proposed method, the rows of normalized Riemann matrix (A) [28] are selected as the phase sequence vector [29] in the SLM technique for PAPR reduction [28], [29].The Riemann matrix (A)of the size NxN is obtained by removing the first row and first column of matrix $(\mathrm{R})$ of size $(\mathrm{N}+1) \mathrm{X}(\mathrm{N}+1)$ [30]. Where

$$
\begin{aligned}
& R(i, j)=i-1 \text { if } i \text { divides } j \\
& =-1 \text { otherwise }
\end{aligned}
$$

The elements ofr ${ }^{\text {th }}$ row in Riemann matrix (A) are either $r$ or $-1,1<\mathrm{r}<\mathrm{N}$. The $\mathrm{r}^{\text {th }}$ row of Riemann matrix (A) is given as:

$$
\mathrm{c}=\lfloor\mathrm{N} / \mathrm{i}+1\rfloor
$$

where c represents number of elements having value rand remaining elements are -1 ,L.Jis greatest integer function. Therefore row $(2<\mathrm{r}<\mathrm{U})$ of matrix $\mathrm{A}$ is used as phase sequence $\mathrm{B}^{\mathrm{U}}$.It results in phase change and also 
amplitude change of the modulated data symbols. Therefore the average power of OFDM signals, $\mathrm{X}^{\mathrm{U}}, 1 \leq \mathrm{u} \leq \mathrm{U}$ will not be the same as that of original signal X [30].

For 8x8 Riemann matrix for OFDM-SLM system with 8 subcarriers which is given by:

$R=\left[\begin{array}{cccccccc}1 & -1 & 1 & -1 & 1 & -1 & 1 & -1 \\ -1 & 2 & -1 & -1 & 2 & -1 & -1 & 2 \\ -1 & -1 & 3 & -1 & -1 & -1 & 3 & -1 \\ -1 & -1 & -1 & 4 & -1 & -1 & -1 & -1 \\ -1 & -1 & -1 & -1 & 5 & -1 & -1 & -1 \\ -1 & -1 & -1 & -1 & -1 & 6 & -1 & -1 \\ -1 & -1 & -1 & -1 & -1 & -1 & 7 & -1 \\ -1 & -1 & -1 & -1 & -1 & -1 & -1 & 8\end{array}\right]$

The above matrix shows that, it provides eight phase sequences for PAPR reduction of the OFDM signal. Any one row except first row can be used as phase sequence. In proposed technique, third row is used for phase sequencing in SLM. Value of all elements of third row are -1 except third and seventh element with a value 3 . Hencethe matrix has exactly $c=2$ number of elements with value 3 . In Riemann matrix the elements with value -1 are used as phase factor and they only produce the phase change. The elements other than -1 in the matrix with value 3 produce the amplitude change. The power level of two data subcarriers therefore increases by a factor of 9.This causes the increase in the average energy of the OFDM signal and thus reduces the PAPR. It is therefore evident that whenever any row is used, except the first the row, the PAPR reduces in SLM-OFDM system. The reduction in PAPR is caused by the increase in average energy of the signal after multiplication with phase sequence [31].

\subsection{Wavelet Packet Modulation}

Wavelet Packet Modulation (WPM) is an extension of standard wavelet which represents generalized multi resolution analysis. It uses all subband decomposition i.e. both the higher and lower subbands. The construction of wavelet packet is done using Quadrature Mirror Filter (QMF) pairs of halfband high pass filter and half-band low pass filter $h(n)$ and $g(n)$ respectively of length L. Relation between these filters [32], [33] is represented by

$$
\mathrm{g}[\mathrm{L}-1-\mathrm{n}]=(-1)^{\mathrm{n}} \mathrm{h}[\mathrm{n}
$$

Also they have adjoints or duals which are their complex conjugate time reversed variants given by

$$
h^{\prime}[n]=h *[-n] \text { and } g^{\prime}[n]=g^{*}[-n]
$$

In order to define the sequence of basis f unction $\varphi(t)$ called as wavelet packets, the QMFs $h(n)$ and $\mathrm{g}(\mathrm{n})$ filters are recursively used [34].

$$
\begin{aligned}
& \varphi_{k+1}^{2 r}(t)=\sqrt{2 \sum_{n} h(n) \varphi_{\mathrm{k}}^{\mathrm{r}}(2 \mathrm{t}-\mathrm{n})} \\
& \varphi_{k+1}^{2 r+1}(t)=\sqrt{2 \sum_{n} g(n) \varphi_{k}^{r}(2 t-n)}
\end{aligned}
$$

Following are the orthogonality properties of Wavelet packets which are shown as

$$
\begin{aligned}
& <\varphi_{n}(t-j), \varphi_{n}(t-k)>=\delta(j-k) \\
& <\varphi_{2 n}(t-j), \varphi_{2 n+1}(t-k)>=\delta(j-k)
\end{aligned}
$$

Where <,> is the inner product of the function and $\delta($.$) is the delta function. The analysis pair \left\{\left[\mathrm{h}^{\prime}[\mathrm{n}], \mathrm{g}^{\prime}[\mathrm{n}]\right\}\right.$ generates the wavelet packet carriers whereas the synthesis filter pair generates wavelet packet carriers duals for demodulation. The filters also satisfy the conditions for perfect reconstruction having the magnitude responses as $H(\omega), G(\omega), H^{\prime}(\omega)$ and $G^{\prime}(\omega)$ [34].

$$
\begin{aligned}
& H^{*}(\omega+\pi) H^{\prime}(\omega)+G^{*}(\omega+\pi) G^{\prime}(\omega)=0 \\
& H^{*}(\omega) H^{\prime}(\omega)+G^{*}(\omega) G^{\prime}(\omega)=0
\end{aligned}
$$


In (8) and (9) the the superscript $\mathrm{r}$ represents index of the carrier at any given depth of the tree $\mathrm{k}$. Let $M$ be the number of WPM subcarriers which are derived from $k$ iterations and is given by $M=2^{K}$. After the WPM operation, the ouput signal is a linear combination of data symbols $\mathrm{a}_{\mathrm{u}, \mathrm{l}}$ of parallel branches, wavelet packet bases and data index $\mathrm{k}$ is represented as follows:

$$
x[n]=\sum_{u} \sum_{l=0}^{M-1} a_{u, l} \varphi_{k}^{r}(n-u M)
$$

With the help of duals of these bases, the data is demodulated at the receiver. The Inverse Discete Wavelet Packet Transform (IDWPT) process is carriedout at transmitter of side of the system and Discete Wavelet Packet Transform (DWPT) process at receiver side [35].

\subsection{The Proposed System}

WPM based, SLM technique with Riemann Matrix is proposed here for PAPR reduction. Block diagram representation is given in Figure 2. The input bit streams from source are first converted to constellation mapping (BPSK/QPSK) N stream of symbols $\{\mathrm{x}(\mathrm{k})\}$ they are then replicated to get L finite number of parallel branches. These $\mathrm{L}$ parallel branches carrying the same information are multiplied by phase sequences generated from normalized Riemann matrix. Then WPM operation is carried out on these modified data and the WPM frame that has least PAPR is selected for transmission.

The k different WPM frames mathematically represented as $x_{[n]}^{k}$.

They are obtained by subcarrier wise multiplication with the rows of the Riemann matrix $r_{l}^{k}$.

Here $1=(1,2, \ldots \mathrm{L})$ represents index of the frame and $\mathrm{k}=(1,2, \ldots \mathrm{N})$ represents subcarrier index. The L different WPM frames obtained after multiplication of subcarriers with rows of Riemann matrix which represents binary phase sequence are shown as:

$$
\mathrm{X}_{\mathrm{L}}[\mathrm{k}]=\mathrm{X}[\mathrm{k}] * \mathrm{r}_{1}[\mathrm{k}]
$$

These modified data blocks are then transformed by IDWPT operation and the peak-to-average power ratio of this transformed data is calculated. The output of IDWPT block is represented as

$$
\mathrm{x}_{1}[\mathrm{n}]=\operatorname{IDWPT}\left(\mathrm{x}_{1}[\mathrm{k}]\right)
$$

The one with minimum is selected for transmission and is represented as:

$$
\bar{l}=\operatorname{argmin}_{1 \leq l \leq L}\left(P A P R\left(x_{l}\right)\right)
$$

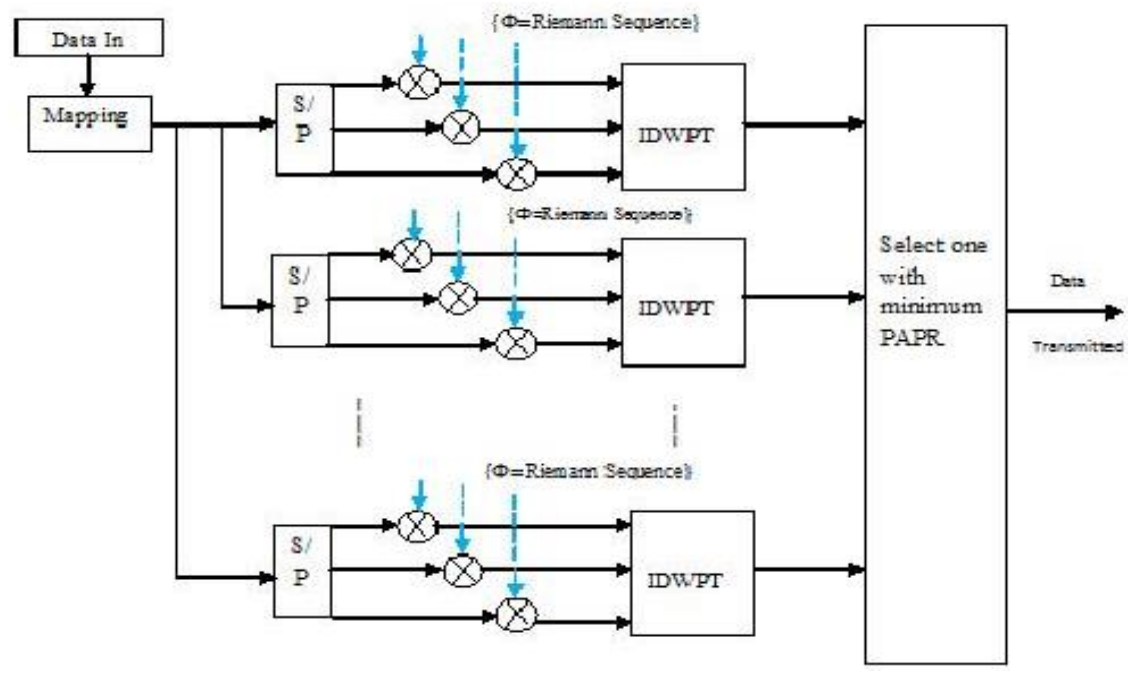

Figure 2. Proposed transmitter functional block diagram 


\section{RESULTS AND ANALYSIS}

Computer simulation using MATLAB software is carried out to analyze the performance of various Combinations: OFDM-classical SLM (using Random sequence), OFDM-SLM with Riemann sequence. The performance analysis of WPM based classical SLM, WPM based SLM with Riemann sequence (proposed system) is done. Performance metric used is Complementary Cumulative Distribution Function (CCDF), which is calculated at the clip rate of $10^{3}$ random OFDM symbols. The value of the phase rotation factors for classical SLM is $+1,-1,+\mathrm{j},-\mathrm{j}$. The simulation parameters are stated in Table 1 .

Table 1. Simulation Parameters

\begin{tabular}{lc}
\hline System parameters & Value \\
\hline Number of subcarriers(N) & $64,128,256,512$ \\
Modulation type & QPSK \\
Phase rotations for classical SLM & $(1, j,-1,-j)$ \\
Sub block size $(\mathrm{K})$ & 8 \\
Wavelet family & Daubechies,coiflet, symlet and bior \\
\hline
\end{tabular}

CCDF of PAPR is plotted for parameters given in Table 1, for all combinations of OFDM, WPM, SLM, and Riemannsequence Performance evaluation of all possible combinations is carried out.

\subsection{Original OFDM and WPM for Different Values of Sub-carriers (N)}

Figure 3 shows the PAPR performance in form of CCDF using OFDM and WPM. Performance of both multicarrier modulation systems is carried out for $\mathrm{N}=64,128,256$ and 512. Comparatively, PAPR reduction using WPM is better but as number of subcarriers increases, PAPR also increases.

\subsection{OFDM, OFDM based Classical SLM and WPM based Classical SLM}

Figure 4 shows the CCDF plots comparing OFDM, OFDM based classical SLM and WPM based classical SLM. Experimentation ensures a good PAPR reduction even though the number of subcarriers is increased from 64 to 512.The WPM based classical SLM shows good PAPR reduction as compared to OFDM with classical Smothered is a PAPR value of 7dB for OFDM based classical SLM system and 6.4 dB for WPM based classical SLM 64 number of subcarriers. There is an improvement of PAPR reduction from $0.6 \mathrm{~dB}$ to $1 \mathrm{~dB}$ from 64 to 512 subcarriers.

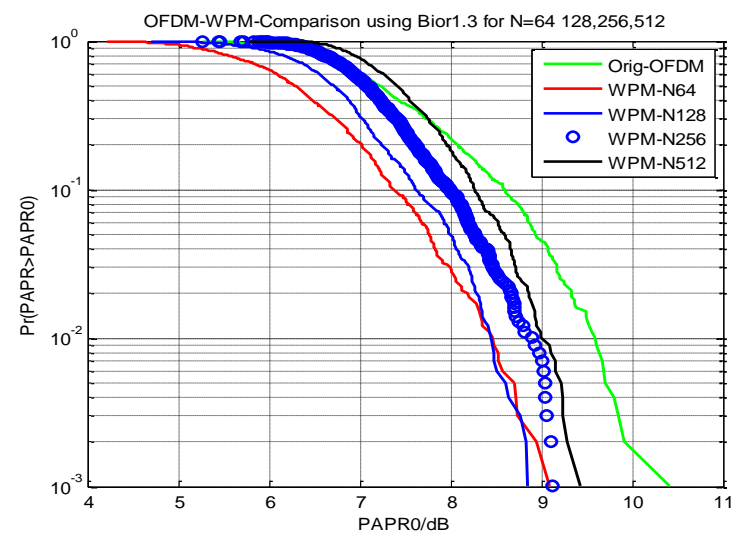

Figure 3. CCDF plot for comparison between OFDM and WPM for $\mathrm{N}=64$

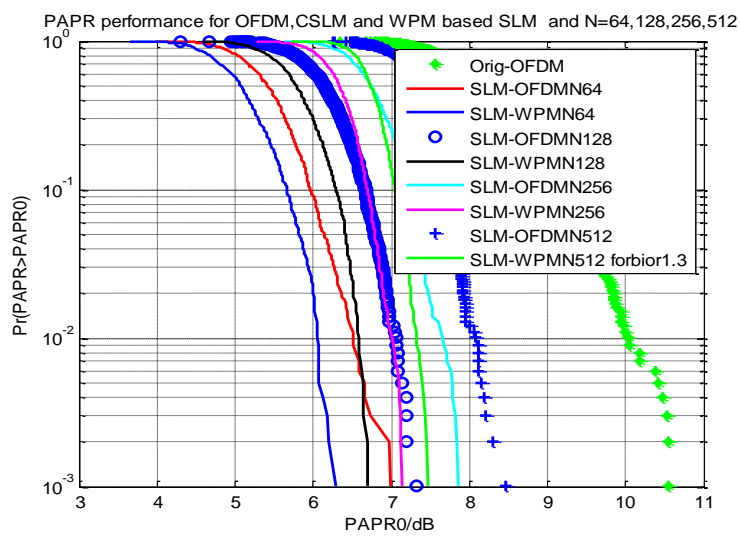

Figure 4. CCDF plot for comparison between OFDMOFDM based classical SLM and WPM based classical SLM for $\mathrm{N}=64$ to 512

\subsection{Comparison between OFDM, OFDM based Classical SLM, OFDM base SLM with Riemann Sequence for Different Values of Subcarriers}

CCDF plots shows reduction in PAPR for OFDM system, OFDM based classical SLM which uses random phase sequence and OFDM based SLM which uses Riemann sequence. Significant reduction in PAPR occurs when Riemann matrix is used for phase rotation. 
Figure 5 to Figure 8 shows CCDF of the PAPR for OFDM system, OFDM-classical SLM which uses random phase sequence and OFDM-SLM- Riemann sequence for various subcarriers N= 64, 128,256 and 512 .

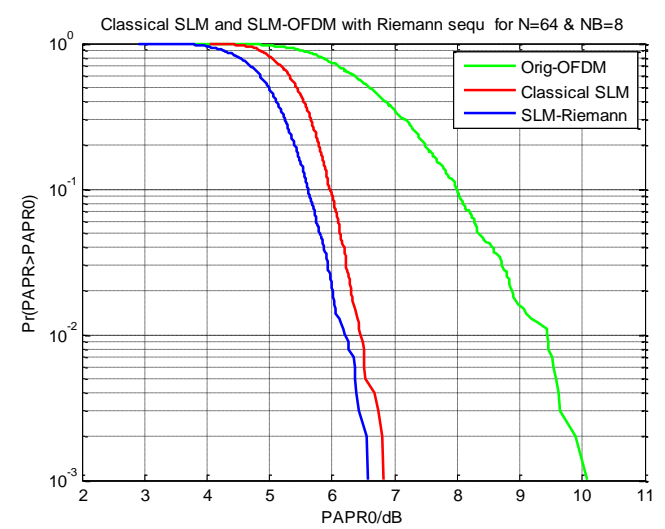

Figure 5. CCDF plot for comparison between OFDM, classical SLM and Riemann sequence based $\mathrm{SLM}$ forN=64

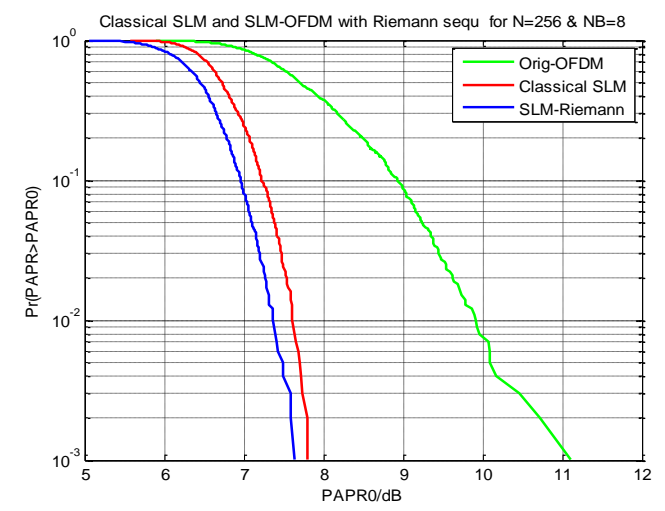

Figure 7. CCDF plot for comparison between OFDM, OFDM based classical SLM and OFDM based SLM with Riemann sequence for $\mathrm{N}=256$

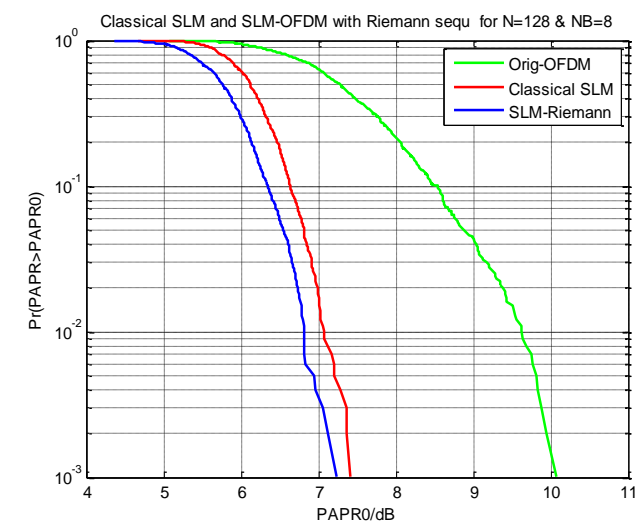

Figure 6. CCDF plot for comparison between OFDM, OFDM based classical SLM and OFDM based SLM with Riemann sequence for $\mathrm{N}=128$

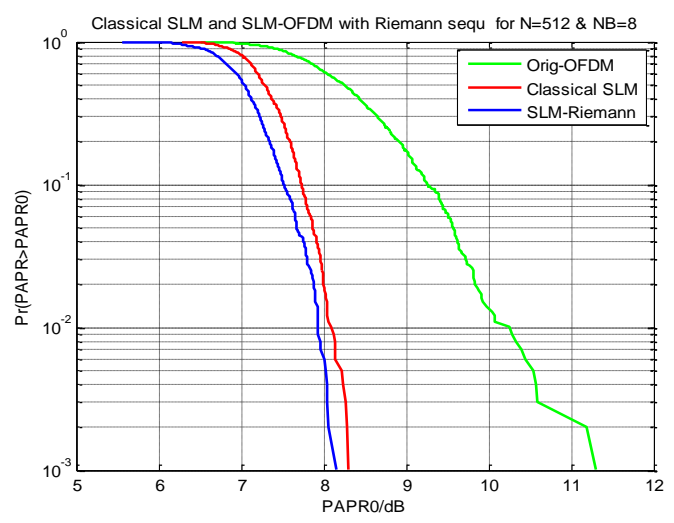

Figure 8. CCDF plot for comparison between OFDM, OFDM based classical SLM and OFDM based SLM with Riemann sequence for $\mathrm{N}=512$

CCDF plot for OFDM, OFDM based classical SLM, OFDM based SLM with Riemann sequence is considered here. Both of them have yielded the notable improvements compared to the OFDM curve and there is also an improvement by Riemann sequence over the random sequence. PAPR reduction in the OFDMbasedSLM with Riemann sequence is improved as compared to OFDM based classical SLM system (using random sequence). There is an improvement of $3.4 \mathrm{~dB}$ to $2 . \mathrm{BdB}$ respectively as compared to OFDM system for different values of number of subcarriers. For the all above figures the effect of increasing number of subcarriers on PAPR reduction is presented. As $\mathrm{N}$ increases the PAPR value also increases because the peak of the signal is $\mathrm{N}$ times the average power if all the sub-carriers are of the same phase.

\subsection{Effect of Riemann Sequence and Performance Evaluation of Proposed System}

Performance evaluation of proposed system which is WPM based SLM with Riemann matrix is presented in Figure 9.It is also compared with all possible combinations i.e.OFDM, OFDM based classical SLM, OFDM based SLM with Riemann sequence, WPM based classical SLM, WPM based SLM with Riemann sequence. The proposed system gives minimum PAPR among all. Improvement in PAPR reduction 
is considerably noticeable in the proposed system as compared other systems mentioned. The proposed system has shown the PAPR reduction of $5 \mathrm{~dB}$ as compared to OFDM system as against $3 \mathrm{~dB}, 3.2 \mathrm{~dB}$ and 3.2dB of classical SLM, Riemann based SLM and WPM based SLM as compared to OFDM system. Number of subcarriers used are 128 and wavelet used is bior 1.3. The factors which are contributing to PAPR reduction in proposed system as compared to OFDM are SLM technique, Riemann matrix and wavelet packet modulation. The SLM being the probabilistic based technique has an advantage of statistically improving characteristics of PAPR distribution without signal distortions. The Riemann matrix is the deterministic matrix which has real valued elements. These real valued elements significantly increase the average power of the signal. And wavelet packet modulation uses orthogonal wavelet packet bases which in turn allow to change the shapes of the subcarrier by changing the properties of filter banks.

\subsection{Impact of Choice of Wavelets on the System}

Proposed system with various wavelets is presented in Figure 10. The CCDF plots of OFDM, OFDM based classical SLM, OFDM based SLM with Riemann sequence and proposed system for different wavelets is shown and the effect of using various wavelets on PAPR reduction is also visible. Proposed system with different wavelets show the improvement over all conventional systems. Various wavelet families considered are Daubechies db3, coiflet1, symlet3, bior 1.3 (all of length 6) used for analysis and bior 1.3 is gives minimum PAPR.

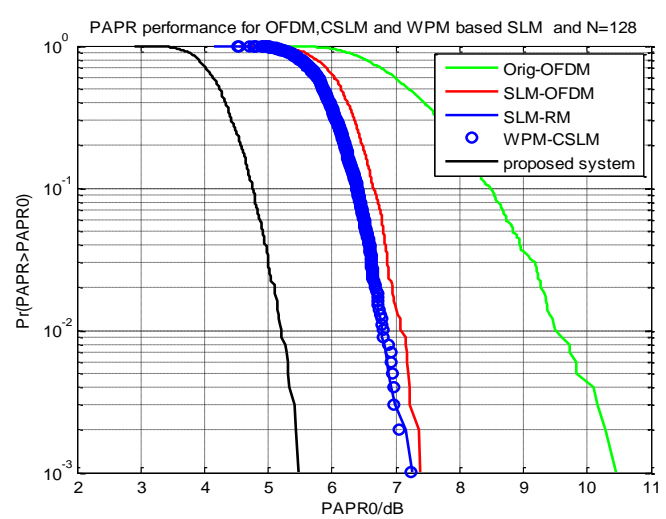

Figure 9. CCDF of the PAPR for comparison between OFDM, OFDM based classical SLM, OFDM based SLM with Riemann sequence, WPM based classical SLM and proposed system, (WPM based SLM with Riemann sequence) for $\mathrm{N}=128$

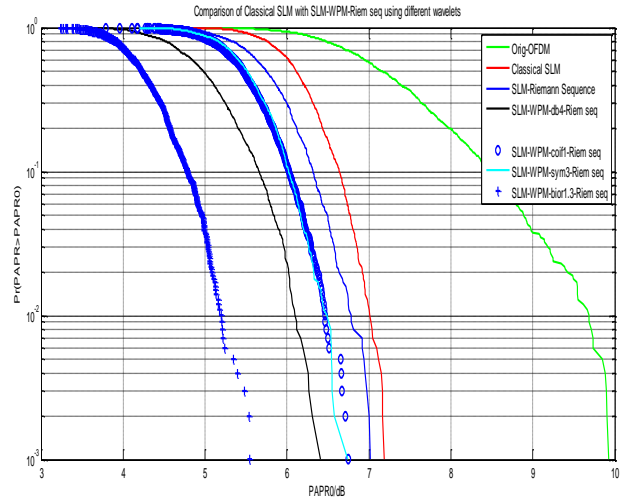

Figure 10. CCDF of the PAPR for comparison between OFDM, OFDM based classical SLM, OFDM based SLM with Riemann sequence and proposed system for different wavelets

\section{CONCLUSIONS}

A PAPR reduction method is proposed in this paper. It uses combination of WPM as multicarrier modulation and Riemann Matrix for phase rotation in SLM which is major contribution of this work. Individually, all technique contribute to PAPR reduction: Wavelets have good orthogonal property in time and frequency localization, SLM is distortionless technique so provides low PAPR, Riemann Matrix for phase rotation in SLM results in increase in average energy of the signal which reduces PAPR. Changing the phases of the subcarriers byRiemann sequence provides different WPM frames with different PAPR. Out of these frames, one WPM frame with minimum PAPR will be selected for transmission. Proposed technique is compared with various combinations of OFDM, WPM, Random and Riemann Matrix for phase rotation in SLM. It is numerically confirmed from results that.

PAPR reduction is better when WPM is used as multi carrier modulation than OFDM. Confirmation of this conclusion continues with additional efforts of introducing classical SLM, i.e. random phase rotation in SLM. An attempt of using Riemann matrix for phase rotation in SLM is proved to be better than random phase rotation; evenwith OFDM is used as multi carrier modulation. With proposed technique, i.e. using combination of WPM as multi carrier modulation and Riemann matrix for phase rotation in SLM, PAPR reduction overpowers all findings. Finally, irrespective of all techniques, as $\mathrm{N}$, number of subcarriers increase, PAPR increases. The importance of this method lies in its easy to implementation. 


\section{REFERENCES}

[1] S.Han and J.Lee, "An Overview of Peak-to-Average Power Ratio Reduction Techniques for Multicarrier Transmission”, IEEE Wireless Communication, 2005, vol.12, no.2, 56-65.

[2] R.V.Nee and R.Prasad, "OFDM for wireless multimedia communications", Boston, London: Artech House, 2000:11-15.

[3] Hajar Abdelali, Smail Bachir and Mohamed Oumsis, "New Technique Combining the Tone Reservation Method with Clipping Technique to Reduce the Peak-to-Average Power Ratio", International Journal of Electrical and Computer Engineering, Dec.2018, vol.8, no.6, 5215-226.

[4] Md.M. HasanandS.S.Singh, "PAPR analysis of FFT and wavelet based OFDM systems for wireless Communications"' IJCA Journal, Dec. 2012; vol.60, vol.15, 38-42.

[5] X.Li and L.J.Cimini, Jr., "Effect of Clipping and Filtering on the Performance of OFDM", IEEE Communication Letters, May 1998,vol. 2, no.5, 131-33.

[6] Kelvin Anoh, CagriTanriover, B.Adebisi, "On the Optimization of Iterative Clipping and Filtering for PAPR Reduction in OFDM Systems", IEEE Access, June 2017, vol 5, 12004-12013.

[7] A.E.Jones, T. A. Wilkinson and S.K.Barto, "Block Coding Scheme for Reduction of Peak to Mean Envelope Power Ratio of Multicarrier Transmission Scheme", Elect. Lett., Dec. 1994; vol.30, no.25, 2098-2099.

[8] B.S.Krongold and D.L.Jones, "PAR Reduction in OFDM via Active Constellation Extension", IEEE Transactions on Broadcasting, Sept. 2003, vol.49, no.3, 258-68.

[9] S.H.Muller and J.B.Huber, "OFDM with Reduced Peak-to-Average Power Ratio by Optimum Combination of Partial Transmit Sequences”, Elect. Lett., Feb. 1997, vol.33, no.5, 368-69.

[10] R.W.Bauml, R.F.H.Fisher and J.B.Huber, "Reducing the Peak-to-Average Power Ratio of Multicarrier Modulation by Selected Mapping", Elect. Lett.,Oct. 1996, vol.32,no.22, 2056-57.

[11] G.R.Hill, M.Faulkner and J.Singh, "Reducing the Peak-to-Average Power Ratio in OFDM by Cyclically Shifting Partial Transmit Sequences", Elect. Lett.,Mar. 2000, vol.36,no.6, 560-61.

[12] Lei Guan and AndingZhu.Gaussian, "Pulse-Based Two-Threshold Parallel Scaling Tone Reservation for PAPR Reduction of OFDM Signals", International Journal of DigitalMultimedia Broadcasting, Volume 2011, Article ID 470310, 9 pages.

[13] AzlineIdris,et al. PAPR Reduction Using Huffman and Arithmetic Coding, "Technique in F-OFDM System”, Bulletin of Electrical Engineering \& Informatics, June 2018, vol. 7, no.2, pp.257-263.

[14] HocinAit-Saadi, Jean-Yves Chouinard and AbdarrazasGuessaum, "A PAPR Reduction for OFDM signals Based on Self Adaptive Multiplication DE Algorithm”, International Journal of Electrical and Computer Engineering (IJECE), Oct 2017, vol.7, no.5, pp.2651-2660.

[15] N.Prabha, K.M.Ravikumar, "PAPR Reduction at Large Multi-User-MIMO-OFDM using Adaptive Data Detection Algorithm", Indonesian Journal of Electrical Engineering and Computer Science. Dec 2018, vol.12, no.3, pp.1071-1080.

[16] A.B.Aicha, S.B. Jebara, "PAPR Analysis and Reduction in WPDM", $1^{\text {st }}$ International Symposium on Control, Communication and Signal Processing, Hmmermet, Tunisia, 2004, pp 315-318.

[17] M.Gautier, C. Lereau, Marylin Arndt, J. Lienard, "PAPR Analysis in Wavelet Packet Modulation', $3{ }^{\text {rd }}$ International Symposium on Control,Communication and Signal Processing (ISCCSP),St.Julians,Malta,2008.

[18] Volkan Kumbasar, O־guzKucur, "Better wavelet packet tree structures for PAPR reduction in WOFDM systems', Digital Signal Processing,Elsevier, 2008, vol.18, no.6, 885-891.

[19] M.Rostamzadeh, V. T. Vakily, "PAPR Reduction in Wavelet Packet Modulation", $5^{\text {th }}$ IEEE International MultiConference on Systems, Signals and Devices, Amman, Jordan, 2008.

[20] Mohan Baro,JacekIIow, "PAPR Reduction in OFDM Using Wavelet Pre-processing", 5 ${ }^{\text {th }} I E E E$ Consumer Communications and Networking Conference (CCNC),Las Vegas, NV, USA, 2008,195-199.

[21] Berna Torun, M.K.Lakshmanan and H. Nikookar, "Peak-to-Average Power Ratio Reduction of Wavelet Packet Modulation by Adaptive Phase Selection", $21^{\text {st }}$ Annual IEEE International Symposium on Personal Indoor and Mobile Radio Communications, Istanbul, Turkey, 2010, 105-110.

[22] M.N.Suma, S. V. Narsimhan, B. Kanmani, "Orthogonal Frequency Division Multiplexing Peak-to-Average Power Ratio Reduction by Best Tree Selection Using Coded Discrete Cosine Harmonic Wavelet Packet Transform", IET Communications Journal, 2014, vol.8, no.11, 1875-1882.

[23] J.Zakaria, MohdFadzil, MohdSale, "PAPRReductionScheme: Wavelet Packet Based PTS with Embedded Side Information Data Scheme”, IET Communications Journal, 2016, vol.11, no.1, 127-135.

[24] Jayalath, A.D.S., Tellambura, C., and Wu, H., "Reduced complexity PTS and new phase sequences for SLM to reduce PAP of an OFDM signal”, VTC, Tokyo, Japan, 2000, vol.3, 1914-1917.

[25] Lim, D.W., et al., "On the phase sequence set of SLM OFDM scheme for a crest factor reduction", IEEE Trans. Signal Process., 2006, vol. 54, no.5, 1931-1935.

[26] Cheng, P., Xiao, Y., Dan, L., and Li, S., "Improved SLM for PAPR reduction in OFDM system", PIMRC, Athens, Greece, 2007, 1-5.

[27] J.A.C. Bingham, "Multicarrier Modulation for Data Transmission: An idea Whose Time has Come", IEEE Communications Magazine, 1990, vol. 28, no.5, 5-14.

[28] N.V.Irukulpati, V. K. Chakka and A. Jain, "SLM based PAPR reduction of an OFDM signal using new phase sequence", IEEE Electronics Letters, July, 2009. 
[29] V.Sudha, B.Anilkumar, M.S.Samantha, D.Sriramkumar, "A low-complexity modified SLM with new Phase Sequences for PAPR Reduction in OFDM System”, India Conference (INDICON), Dec, 2015, Annual IEEE Conference, $17-20$.

[30] F. Roesler, "Riemann's hypothesis as an eigen value problem Linear Algebra Application", 1986, vol.81, 153-198.

[31] S.Harne, A. Zanjade, "PAPR Reduction in OFDM System using Phase Sequence of Riemann Matrix. International Advanced Research Journal in Science", Engineering and Technology, 2017, vol.7, no.9.

[32] Jamaluddin Zakaria, Mohd. FadzliMohdSalleh, "PAPR reduction scheme: wavelet packet based PTS with embedded side information data scheme", IET Communications.2016, vo.11, no.1, 127-135.

[33] I.Daubechies, "Ten Lectures on Wavelets", SIAM, Philadelphis, 1992.

[34] M. Vetterli and J. Kovacevic, "Wavelets and Subband Coding”, Prentice Hall PTR. Englewood Cliffs, New Jersey, 1995.

[35] S.L.Linfoot, M.K.Ibrahim and M. M. Al-Akaidi, "Orthogonal wavelet division multiplex: an alternative to OFDM", IEEE Trans. on Consumer Elec., May 2007, vol 53, No.2. 278-284. 